\title{
Burnout en deportistas: Prevalencia del síndrome a través de dos medidas
}

\author{
Burnout in athletes: Prevalence of the syndrome through two measures
}

\section{Burnout em atletas: Prevalência da síndrome através de duas medidas}

\author{
De Francisco, C. ${ }^{1}$, Garcés de los Fayos, E.J. ${ }^{2}$, \& Arce, C. ${ }^{3}$ \\ 1 Universidad Católica San Antonio (Murcia), 2 Universidad de Murcia, 3 Universidad de Santiago
}

\begin{abstract}
Resumen: En este trabajo se ha estudiado la prevalencia del síndrome de burnout en una muestra de 442 deportistas y la relación entre padecer el síndrome, o algunas de sus dimensiones, y algunas variables significativas en el ámbito del deporte (género de los deportistas, edad, nivel competitivo y volumen de entrenamiento). Se aplicaron dos instrumentos de medida del burnout en deportistas, el Inventario de Burnout en Deportistas-Revisado (IBD-R) y el Athlete Burnout Questionnaire (ABQ). Para el análisis de datos (estadísticos descriptivos, distribución de frecuencias, pruebas t y análisis de varianza) se utilizó el paquete estadístico SPSS. Se han obtenido porcentajes similares sobre la prevalencia del síndrome con ambos cuestionarios. Cerca de un $4 \%$ padece el síndrome, existiendo ciertas diferencias en relación al género, nivel deportivo y volumen de entrenamiento. Los resultados nos hacen reflexionar acerca de la relevancia que tiene la prevalencia del síndrome en este entorno, y se plantean algunas estrategias de futuro encaminadas a su prevención.

Palabras clave: Burnout, deportistas, prevalencia, IBD-R, ABQ.

Abstract: This work has studied the prevalence of the burnout syndrome in a sample of 442 athletes and the relationship between the syndrome, or some of its dimensions, and some significant variables in the field of sport (athletes gender, age, competitive level, and volume of training). Two instruments of measurement of burnout in athletes, the Burnout Inventory in Athletes (Inventario de Burnout en Deportistas-Revisado, IBD-R) and the Athlete Burnout Questionnaire (ABQ) have been applied. For data
\end{abstract}

analysis (descriptive statistics, frequencies distribution, $\mathrm{t}$ test and analysis of variance) we used the statistical package SPSS. Similar percentages have been obtained on the prevalence of the syndrome with both questionnaires. About $4 \%$ of the athletes suffer from the syndrome, and there are certain differences in relation to gender, competitive level and volume of training. Results make us think about the relevance of its prevalence in sport context and some future strategies for its prevention.

Key words: Burnout, athletes, prevalence, IBD-R, ABQ.

Resumo: Este trabalho estudou a prevalência da síndrome de burnout em uma amostra de 442 atletas e da relaçáo entre a síndrome, ou alguma de suas dimensôes, e algumas variáveis significativas no domínio do desporto (atletas de gênero, idade, nível competitivo, e volume de treinamento). Dois instrumentos de medida de burnout em atletas, o Inventário de Burnout em Atletas (Inventario de Burnout en Deportistas-Revisado, IBD-R) eo atleta Burnout Questionnaire (ABQ) foram aplicadas. Para análise dos dados (estatística descritiva, distribuição de freqüências, teste t e análise de variância) foi utilizado o pacote estatístico SPSS. Percentagens semelhantes foram obtidos sobre a prevalência da síndrome com ambos os questionários. Cerca de $4 \%$ dos atletas sofrem com a síndrome, e há certas diferenças em relação ao sexo, nível competitivo e volume de treinamento. Os resultados fazem-nos pensar sobre a relevância da sua prevalência em contexto desportivo e algumas estratégias futuras para a sua prevenção.

Palabras chave: Burnout, atletas, prevalência, IBD-R, ABQ.

\section{Introducción}

Existen dos factores claramente implicados con la práctica deportiva; de una parte, es uno de los aspectos sociales más demandados y consumidos por la población. $\mathrm{Y}$ de otra, la enorme presión que los deportistas sufren, en tanto que suelen representar las inquietudes, ilusiones, sueños, frustraciones e iras, de un conjunto amplio de seguidores. Quizás por ambos motivos, los deportistas pueden considerarse una población de riesgo en cuanto a su salud psicológica, sobre todo en lo concerniente a síndromes que están estrechamente relacionados con dicha presión mediática, como es el caso del burnout, trastorno que empieza a constituirse como un fenómeno importante en esta población (Garcés de Los Fayos, 2004). Podemos considerar el inicio del estudio del burnout en el deporte con el trabajo de Flipin (1981), si bien hubo

Dirección para correspondencia [Correspodence address]: Cristina De Francisco Palacios. Universidad Católica San Antonio (UCAM). Murcia. E-mail: cdefrancisco@ucam.edu

que esperar algo más para disponer de una definición acorde al contexto. Maslach y Jackson (1981) plantea la definición más aceptada en el ámbito general del burnout como consecuencia de padecer un estrés severo y duradero en el tiempo, apareciendo las tres dimensiones que lo constituyen: agotamiento emocional, despersonalización y reducida realización personal, siendo esta definición la que posteriormente Fender (1989) adaptaría al ámbito deportivo.

Siguiendo el esquema de un trabajo anterior (Garcés de Los Fayos, 1999), un primer nivel de análisis en la investigación ha consistido en conocer aquellas variables que anteceden al problema desde los tres contextos más inmediatos del deportista: social-familiar, deportivo y personal (interno). Parece demostrado que las complejas interacciones de las variables predictoras, su intensidad y frecuencia, así como la percepción más o menos aversiva que el deportista tenga de ellas, provocarán un estado emocional en el deportista que puede facilitar la aparición de burnout (Garcés de Los Fayos, 
Jara y Vives, 2006). Un segundo nivel de análisis se ha dirigido a estudiar las consecuencias que puede ocasionar este trastorno: problemas físicos, insatisfacción con el estilo de vida, insatisfacción con su rol deportivo, expectativas incumplidas, disminución de la diversión, problemas de concentración, negativas sensaciones y componentes afectivos, y sentimientos de aislamiento (Dosil, 2008; Garcés de Los Fayos, Olmedilla y Jara, 2006; Gould, Tuffey, Udry y Loerh, 1996).

De acuerdo con lo anterior, surge una clara preocupación por analizar la prevalencia que el burnout puede tener en los deportistas, habiéndose realizado ya trabajos muy interesantes en países iberoamericanos (Tutte y Garcés de Los Fayos, 2010). A continuación se plantean diferentes datos en estudios realizados en países del mismo entorno sociocultural al nuestro, que complementan los datos más relevantes y actuales en la investigación sobre burnout en el contexto anglosajón que se han propuesto recientemente por parte de Marín, Ortín, Garcés de Los Fayos y Tutte (2013):

- En Cuba, se señala, entre otros aspectos, el deterioro de las relaciones interpersonales que se presenta como una variable predictora, y también el hecho de que el entorno deportivo puede incidir en la presencia de burnout en los deportistas (Medina, 2001; Medina y García, 2002).

- En Uruguay, profundizaron en el burnout tanto con metodología cuantitativa, como cualitativa, poniendo de manifiesto que el burnout aparece como paso previo al abandono deportivo y que el apoyo social podría actuar como variable protectora, pues disminuye la posibilidad de sufrir el síndrome, mientras que las coacciones sociales, aumentan el riesgo de padecerlo (Tutte, 2009; Tutte, Blasco y Cruz, 2006; Tutte y Suero, 2009).

- En México, encuentran que la frecuencia de burnout en deportistas es elevada, destacando que la falta de apoyo familiar parece convertirse en un buen predictor. Además parece existir una cierta relación entre sufrir burnout y padecer un potencial trastorno psicológico, lo que tiene una gran importancia sobre todo, porque se hace necesario encontrar las relaciones que el burnout, como síndrome psicopatológico, posee en el espectro amplio de la psicopatología (Reynaga, 2008, 2009; Reynaga y Pando, 2005).

- En Colombia, Sierra y Abellón (2008) han hallado que los deportistas con burnout muestran mayor cantidad de pensamientos irracionales. Además, el síndrome aparece relacionado negativamente con motivación y autoconfianza.

- En México, Percastre (2009) concluye que aquellos deportistas que muestran algunas de las dimensiones del burnout también destacarían por autoconfianza y autoconcepto más negativos que el resto. Por otra parte, parece que las estrategias de afrontamiento de los deportistas frente a las dimensiones del burnout son variadas, aunque quizás no se muestren muy eficaces.

- En Costa Rica, Hernández (2009) ha constatado el poder aversivo que, en ocasiones, puede tener el ámbito deportivo, por lo que hay que abordar la posibilidad de mejorar dicho contexto, vigilando la influencia negativa que la familia presenta, entre otras cosas, porque no valora en su justa medida las exigencias a las que somete al deportista.

Este trabajo se sitúa en esta realidad descrita, donde nuestro interés se centra en conocer la prevalencia de este trastorno psicopatológico en población deportiva a partir de dos instrumentos de evaluación. En la Universidad de Murcia, se ha desarrollado el Inventario de Burnout en Deportistas (IBD; Garcés de Los Fayos, 1999) posteriormente revisado, simplificado y validado (IBD-R; Garcés de Los Fayos, De Francisco y Arce, 2012). De manera independiente, en la Universidad de Santiago de Compostela, en colaboración con la Universidad de Carolina del Este, se ha realizado una adaptación del Athlete Burnout Questionnaire (ABQ; Raedeke y Smith, 2001) a la cultura española, primero en futbolistas (Arce, De Francisco, Andrade, Arce y Raedeke, 2010) y luego validado en diferentes deportes, tanto colectivos como individuales (Arce, De Francisco, Andrade, Seoane y Raedeke, 2012). No han sido muchos los trabajos que han ofrecido datos sobre la prevalencia del síndrome en esta población, situando ésta en un rango entre el 6 y el 10\% (Garcés de Los Fayos, 1999; Jiménez, Jara y García,1995; Medina y García, 2002), ni se han planteado diferencias claras en función de que el deporte fuera individual o colectivo. Por ello, parece necesario contrastar si los mismos se mantienen en la actualidad ofreciendo datos basados en los dos instrumentos, aspecto que no ha sido abordado hasta la fecha. Además se analiza si existen diferencias en los niveles de burnout por estratos de género, edad, nivel competitivo y volumen de entrenamiento.

\section{Método}

\section{Participantes}

La muestra estuvo formada por 442 deportistas españoles. Un $52.9 \%$ de la muestra eran hombres y un $47.1 \%$ mujeres, oscilando sus edades entre los 14 y los 29 años (media= 18.86; desviación típica $=2.65)$. Un $25.9 \%$ competía a nivel local/comarcal, un $42.2 \%$ en categoría autonómica (nivel competitivo entre local y nacional/internacional) y el resto, $32 \%$ a nivel nacional/ internacional. La muestra se recogió en un total de 34 deportes. De ellos, 10 deportes fueron colectivos: baloncesto (11.1\%), balonmano (2.7\%), fútbol (22.4\%), fútbol sala (3.4\%), hockey (2.7\%), remo $(2.5 \%)$, rugby $(1.4 \%)$, vela $(2 \%)$, voleibol $(534 \%)$ y waterpolo $(0.5 \%)$. Al respecto de las modalidades que se practi- 
caban de manera individual, el número ascendía a 24: atletismo (7.2\%), automovilismo (0.75), bádminton (0.9\%), boxeo $(0.7 \%)$, ciclismo (1.1\%), esgrima (0.5\%), hípica (1.6\%), judo (1.4\%), kárate $(1.8 \%)$, kung-fú $(1.4 \%)$, lucha $(0.7 \%)$, natación $(7 \%)$, patinaje $(2.3 \%)$, piragüismo $(2.3 \%)$, salvamento $(0.7 \%)$, squash (09\%), surf (1.4\%), taekwondo (2.9\%), tenis (5.9\%), tenis de mesa $(0.5 \%)$, tiro con arco $(0.5 \%)$, gimnasia $(1.8 \%)$, kickboxing (1.6\%) y triatlón (0.5\%). De todos ellos, el fútbol se sitúa como el deporte mayoritario (22.4\%). El volumen medio semanal de entrenamientos era de 7.38 horas (desviación típica= 5.16).

\section{Instrumentos}

Se utilizaron dos herramientas para la medición del síndrome de burnout en deportistas, el IBD-R (Garcés et al., 2012) y el ABQ (Arce et al., 2012); además de datos sociodemográficos y deportivos como edad, género, deporte practicado, carga de entrenamiento, etc.

El IBD-R es una versión reducida de 19 ítems del IBD (Garcés de Los Fayos, 1999) que evalúa el burnout en deportistas como un constructo tridimensional, caracterizado por Agotamiento Emocional (AE, 7 ítems, ejemplo de ítem: Llevar una disciplina de trabajo en mi actividad deportiva me agota), Reducida Realización Personal (RRP, 7 ítems, ejemplo de ítem: Soy efectivola tratando los problemas de las personas que me rodean en el ámbito deportivo) y Despersonalización (D, 5 ítems, ejemplo de ítem: Realmente no me importa lo que les ocurra a las personas que me rodean en mi actividad deporti$v a)$. La escala de respuesta que utiliza es de cinco alternativas: Nunca he sentido o pensado esto (1), Alguna vez al año lo he pensado o sentido (2), Alguna vez al mes lo he pensado o sentido (3), Alguna vez a la semana lo he pensado o sentido (4), Pienso $o$ siento esto a diario (5). Para hallar la puntuación total en cada subescala se suman las puntuaciones de los ítems que componen dicha subescala. Cuanto mayor es la puntuación, mayor es el nivel de burnout experimentado por el deportista, excepto en los ítems de Reducida Realización Personal que están formulados en dirección contraria: cuánto menor es la respuesta numérica del sujeto, mayor es el grado de burnout experimentado. Los valores de consistencia interna medida a través de Alpha de Cronbach de cada dimensión se sitúan entre .70 y .75 (Agotamiento Emocional, = .73, Reducida Realización Personal $=.70$ y Despersonalización $=.72$ ).

Por su parte, del $A B Q$ se tomó la adaptación española de 15 ítems realizada por Arce et al. (2012) que también se basa en una conceptualización tridimensional del síndrome, y cuyos componentes son Agotamiento Físico/Emocional (AFE, 5 ítems, ejemplo de ítem: Me desgastan las exigencias físicas y mentales del deporte), Reducida Sensación de Logro (RSL, 5 ítems, ejemplo de ítem: Pienso que no estoy rindiendo a mi nivel real en el deporte) y Devaluación de la Práctica Deportiva (DPD, 5 ítems, ejemplo de ítem: Creo que no me interesa tanto el deporte como antes). La escala de respuesta también es tipo Likert con 5 categorías ordenadas: Casi nunca (1), Pocas veces (2), Algunas veces (3), A menudo (4), Casi siempre (5). Los ítems 1 y 14 pertenecientes a la subescala Reducida Sensación de Logro están formulados en dirección contraria a los demás siendo necesario invertir sus respuestas antes de calcular el total de la subescala. Cuanto mayor es la suma de las puntuaciones en una subescala, mayor es el burnout experimentado. Al respecto de la consistencia interna medida a través de Alpha de Cronbach, los valores que presentan cada dimensión son los siguientes: Agotamiento Físico/Emocional $=.81$, Reducida Sensación de Logro $=.71$ y Devaluación de la Práctica Deportiva $=.77$.

\section{Procedimiento}

Para llevar a cabo el presente estudio, se elaboró un cuadernillo de respuesta con los dos instrumentos, que los deportistas rellenaban generalmente antes de una sesión de entrenamiento habitual de forma colectiva, aceptando participar todos los deportistas seleccionados. Se les informó de la confidencialidad de sus respuestas y de la importancia de que éstas fuesen sinceras, otorgando su consentimiento firmado una vez informados. En el caso de menores de edad, previamente se le había facilitado una autorización con los objetivos del estudio para que fuese firmada por los padres/tutores. Todos recibieron las mismas instrucciones y se les indicó que pidiesen ayuda en el caso de que les resultase confusa la redacción del algún ítem.

\section{Análisis de datos}

A continuación se presentan los resultados obtenidos con el programa informático SPSS (versión 17.0) sobre estadísticos descriptivos, baremos obtenidos de los dos cuestionarios y la comparaciones de grupos (pruebas t y ANOVA) acerca de los niveles de burnout por estratos de género, edad, nivel competitivo y volumen de entrenamiento.

\section{Resultados}

\section{Estadísticos descriptivos}

En el Tabla 1 se muestra la media y la desviación típica para los totales y para cada una de las dimensiones de los dos instrumentos, teniendo en cuenta que las puntuaciones medias oscilan entre 1 y 5 para ambos cuestionarios. Las medias, en general, presentan valores bajos para las dos medidas, tanto en puntuaciones totales (entre 1.99 y 2.18) como por subescalas (entre 1.60 y 2.39). En el caso de las desviaciones típicas, los valores más bajos se presentan en las puntuaciones totales: .44 para el IBD-R y 57 para el ABQ. Los valores de las desviaciones típicas para las distintas subescalas oscilan entre .61 y .85 . 
Tabla 1. Estadísticos descriptivos del IBD-R y del ABQ

\begin{tabular}{llll}
\hline Cuestionario & Subescalas & Media & Desviación típica \\
\hline \multirow{4}{*}{ IBD-R } & Total & 1.99 & 0.44 \\
& AE & 1.87 & 0.61 \\
& RRP & 2.39 & 0.67 \\
& D & 1.60 & 0.67 \\
\hline \multirow{4}{*}{ ABQ } & Total & 2.18 & 0.57 \\
& AFE & 2.21 & 0.77 \\
& RSL & 2.37 & 0.70 \\
& DPD & 1.96 & 0.85 \\
\hline
\end{tabular}

Baremos para IBD-R y ABQ

Con la muestra utilizada en este estudio se ha elaborado una tabla de baremos para cada uno de los cuestionarios, el IBD R (Tabla 2) y el ABQ (Tabla 3). En estas tablas se ofrece la correspondencia entre la puntuación total obtenida en cada subescala, a través de la suma de sus ítems, y el valor de $\mathrm{T}$, cuya media se sitúa en 50 y la desviación típica en 10 .

Con el objetivo de ofrecer resultados sobre el porcentaje de deportistas con burnout, y en base a criterios estadísticos, se propone la siguiente categorización: (1) Bajo riesgo de padecer burnout (deportistas cuya puntuación T sea igual o menor a 50); (2) Riesgo moderado (deportistas con puntuaciones T entre 50 y 60); (3) Alto riesgo (deportistas con puntuaciones T entre 60 y 70), (4) Con burnout (deportistas con puntuaciones $\mathrm{T}$ superiores a 70 ).

Tabla 2. Baremos T del IBD-R

\begin{tabular}{|c|c|c|c|c|}
\hline Puntuación T & $\mathrm{AE}$ & RRP & $\mathrm{D}$ & Puntuación $\mathrm{T}$ \\
\hline 102 & 35 & & & 102 \\
\hline 101 & & & 25 & 101 \\
\hline 100 & & & & 100 \\
\hline 99 & 34 & & & 99 \\
\hline 98 & & & 24 & 98 \\
\hline 97 & 33 & & & 97 \\
\hline 96 & & & & 96 \\
\hline 95 & 32 & & 23 & 95 \\
\hline 94 & & & & 94 \\
\hline 93 & & & & 93 \\
\hline 92 & 31 & & 22 & 92 \\
\hline 91 & & & & 91 \\
\hline 90 & 30 & & & 90 \\
\hline 89 & & 35 & 21 & 89 \\
\hline 88 & & & & 88 \\
\hline
\end{tabular}

87

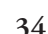

61

60

59

$\begin{array}{lllll}61 & & 22 & & 61 \\ 60 & 17 & 21 & 11 & 50 \\ 59 & & & & 58 \\ 58 & 16 & 20 & & 57 \\ 57 & & & 10 & 56 \\ 56 & 15 & 19 & & 55 \\ 55 & & & & 54 \\ 54 & & 18 & 9 & 53 \\ 53 & 14 & & & 52 \\ 52 & & 17 & & 51 \\ 51 & 13 & & 8 & 50 \\ 50 & & & & 49 \\ 49 & & 16 & & 48 \\ 48 & 12 & & 7 & 47 \\ 47 & & 15 & & 46 \\ 46 & & & & 45 \\ 45 & & 14 & 6 & 44\end{array}$




\begin{tabular}{llll}
43 & 10 & & 43 \\
42 & & 13 & 42 \\
41 & & & 41 \\
40 & 9 & 12 & 40 \\
39 & & & 39 \\
38 & 8 & 11 & 38 \\
37 & & & 37 \\
36 & 7 & 10 & 36 \\
35 & & & 35 \\
34 & & 34 \\
33 & & 33 \\
32 & & & 32 \\
31 & & & 31 \\
30 & & 8 & 29 \\
29 & & & \\
\hline
\end{tabular}

Tabla 3. Baremos T del ABQ.

\begin{tabular}{|c|c|c|c|c|}
\hline Puntuación $T$ & AFE & RSL & DPD & Puntuación T \\
\hline 87 & & 25 & & 87 \\
\hline 86 & 25 & & 25 & 86 \\
\hline 85 & & & & 85 \\
\hline 84 & 24 & 24 & & 84 \\
\hline 83 & & & 24 & 83 \\
\hline 82 & & 23 & & 82 \\
\hline 81 & 23 & & 23 & 81 \\
\hline 80 & & & & 80 \\
\hline 79 & 22 & 22 & 22 & 79 \\
\hline 78 & & & & 78 \\
\hline 77 & & & & 77 \\
\hline 76 & 21 & 21 & 21 & 76 \\
\hline 75 & & & & 75 \\
\hline 74 & & & 20 & 74 \\
\hline 73 & 20 & 20 & & 73 \\
\hline 72 & & & 19 & 72 \\
\hline 71 & 19 & & & 71 \\
\hline 70 & & 19 & & 70 \\
\hline 69 & & & 18 & 69 \\
\hline 68 & 18 & & & 68 \\
\hline 67 & & 18 & 17 & 67 \\
\hline 66 & 17 & & & 66 \\
\hline 65 & & 17 & 16 & 65 \\
\hline 64 & & & & 64 \\
\hline
\end{tabular}

\begin{tabular}{|c|c|c|c|c|}
\hline 63 & 16 & & & 63 \\
\hline 62 & & 16 & 15 & 62 \\
\hline 61 & & & & 61 \\
\hline 60 & 15 & & 14 & 60 \\
\hline 59 & & 15 & & 59 \\
\hline 58 & 14 & & 13 & 58 \\
\hline 57 & & & & 57 \\
\hline 56 & & 14 & & 56 \\
\hline 55 & 13 & & 12 & 55 \\
\hline 54 & & & & 54 \\
\hline 53 & 12 & 13 & 11 & 53 \\
\hline 52 & & & & 52 \\
\hline 51 & & & 10 & 51 \\
\hline 50 & 11 & 12 & & 50 \\
\hline 49 & & & & 49 \\
\hline 48 & & 11 & 9 & 48 \\
\hline 47 & 10 & & & 47 \\
\hline 46 & & & 8 & 46 \\
\hline 45 & 9 & 10 & & 45 \\
\hline 44 & & & & 44 \\
\hline 43 & & & 7 & 43 \\
\hline 42 & 8 & 9 & & 42 \\
\hline 41 & & & 6 & 41 \\
\hline 40 & & & & 40 \\
\hline 39 & 7 & 8 & 5 & 39 \\
\hline 38 & & & & 38 \\
\hline 37 & 6 & & & 37 \\
\hline 36 & & 7 & & 36 \\
\hline 35 & & & & 35 \\
\hline 34 & 5 & & & 34 \\
\hline 33 & & 6 & & 33 \\
\hline 32 & & & & 32 \\
\hline 31 & & & & 31 \\
\hline 30 & & 5 & & 30 \\
\hline
\end{tabular}

Prevalencia del síndrome de burnout en deportistas

Se han obtenido resultados similares sobre la prevalencia del síndrome (operacionalizada ésta a través de porcentajes) con ambos cuestionarios tanto a nivel global, como por subescalas (Figura 1). Alrededor de un 55\% de los deportistas presentan bajo riesgo de padecer burnout $(55.7 \%$ según los datos del IBD-R y el $55.4 \%$ según el ABQ), frente a un 3.8-3.4\% de deportistas que presentan el síndrome (datos del IBD-R y $A B Q$, respectivamente). El porcentaje restante se reparte 
en riesgo moderado $(27.8 \%$ y $28.7 \%)$ y alto riesgo $(12.7 \%$ y $12.4 \%)$.

Analizando los resultados ofrecidos para cada uno de los instrumentos por subescalas, observamos que según el IBD$\mathrm{R}$, un $4.1 \%$ de los deportistas presentarían Agotamiento Emocional, un 2.9\% Reducida Realización Personal y un 5.9\% Despersonalización. Los resultados se replican con los datos recogidos mediante el ABQ ya que hay un $4.3 \%$ de los deportistas con Agotamiento Emocional, un 3.8\% con
Reducida Sensación de Logro y un 5.2\% con Devaluación de la Práctica Deportiva. En ambos instrumentos constatamos que hay un mayor porcentaje de deportistas con puntuaciones altas en las dimensiones que evalúan distancia emocional: Despersonalización (IBD-R) con un 5.9\% (distancia emocional hacia las personas del entorno deportivo) y Devaluación de la Práctica Deportiva (ABQ) con un 5.2\% (distancia emocional hacia el propio deporte).

Figura 1. Prevalencia del burnout medido con el IBD-R y el ABQ

Prevalencia del burnout medida con el IBD-R y el ABQ

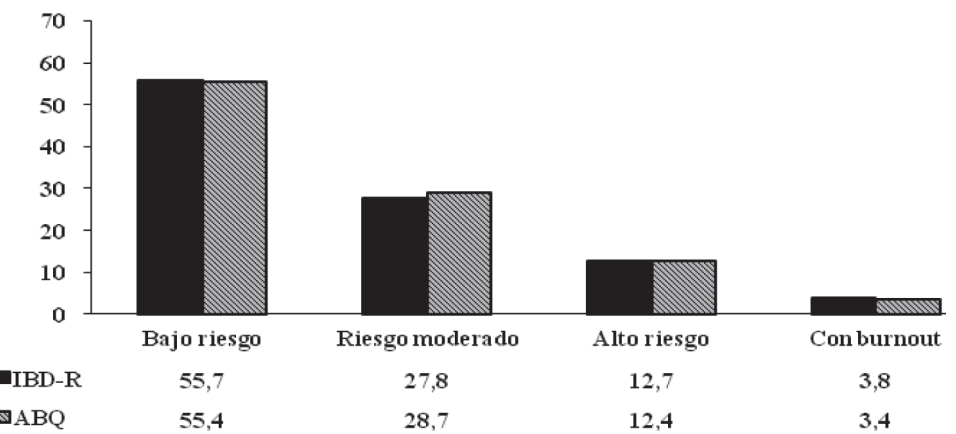

Prevalencia de las subescalas del IBD-R

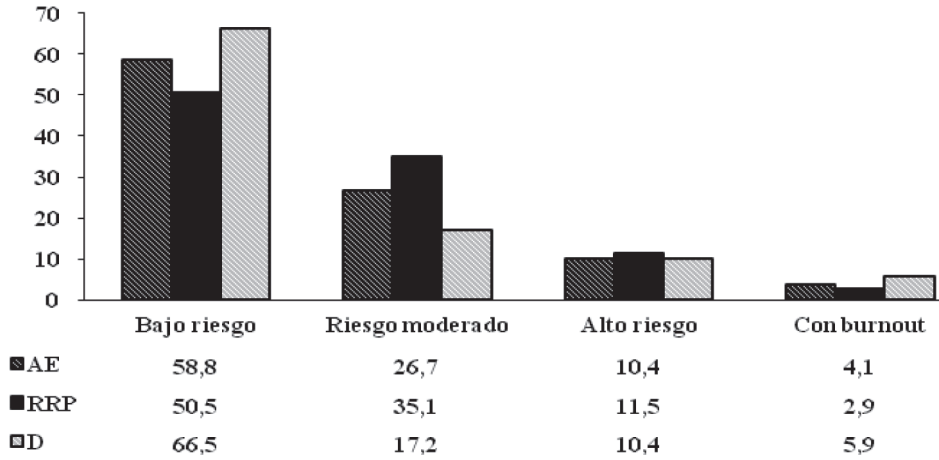

Prevalencia de las subescalas del $\mathrm{ABQ}$

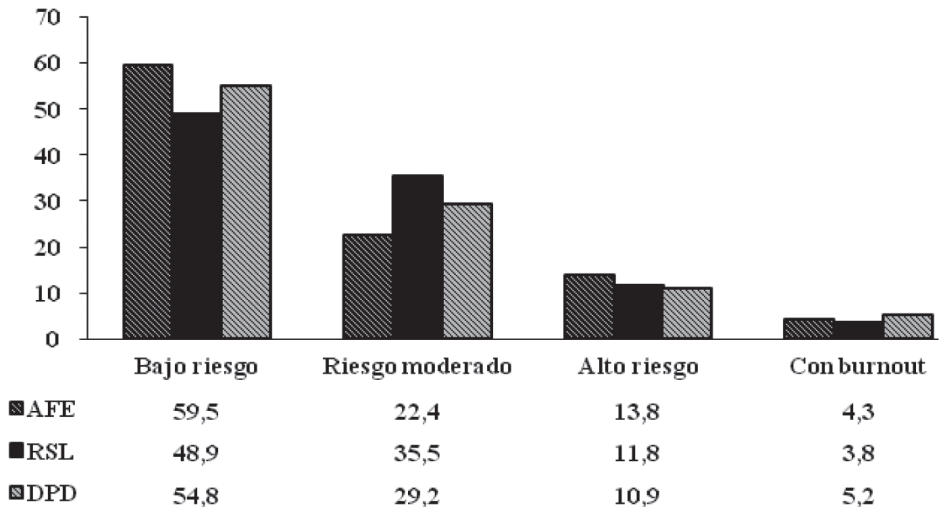


Una vez valorado el porcentaje del síndrome en el conjunto de la muestra, nos interesaba también conocer si existen o no diferencias en los niveles de burnout por estratos de género, edad, nivel competitivo y volumen de entrenamiento.

\section{Burnout, género y edad}

Burnout y género. No se han encontrado diferencias estadísticamente significativas entre hombres y mujeres en la puntuación total de burnout en ninguno de los dos cuestionarios utilizados (IBD-R: $\mathrm{t}=-0.27 ; \mathrm{p}>.05$; $\mathrm{ABQ}: \mathrm{t}=-1.19 ; \mathrm{p}>.05$ ). No obstante, sí se han detectado diferencias en dos subescalas del IBD-R. En Agotamiento Emocional presentan las mujeres una media significativamente más alta que los hombres (1.96 y 1.78 respectivamente; $\mathrm{t}=-3.13 ; \mathrm{p}<.05)$, mientras que en Despersonalización son los hombres los que puntúan más alto que las mujeres (1.70 y 1.50 , respectivamente; $\mathrm{t}=3.22$; $\mathrm{p}<.05)$.

Burnout y edad de los deportistas. Se ha dividido la muestra en dos grupos de edad, correspondientes aproximadamente con grupos de deportistas en formación (14-18 años) y senior (19-29 años) no encontrándose diferencias estadísticamente significativas ( $\mathrm{p}>.05)$, ni en las puntuaciones totales ni en ninguna de las subescalas.

\section{Burnout, nivel competitivo y volumen de entrenamiento}

Burnout y nivel competitivo. Se establecieron tres niveles competitivos: (1) deportistas que competían a nivel local/comarcal, (2) deportistas que competían a nivel autonómico, y (3) deportistas que competían a nivel nacional/internacional. No se han obtenido diferencias estadísticamente significativas para los totales de ninguno de los dos cuestionarios, pero sí en dos de sus subescalas: Reducida Realización Personal (RRP en IBD-R) y Reducida Sensación de Logro (RSL en $A B Q)$. Se realizaron comparaciones a posteriori mediante la prueba de Scheffé (Tabla 4) encontrándose que los deportistas que competían a nivel nacional/internacional presentan una media significativamente más baja en RRP que los que lo hacían a nivel local/comarcal (diferencia entre medias = -0.21 ; $\mathrm{p}<.05$ ). Con respecto a RSL se observa una tendencia parecida ya que el grupo nacional/internacional muestra una media significativamente más baja que el grupo local/comarcal (diferencia de medias $=-.30 ; \mathrm{p}<.05$ ) y autonómico (diferencia de medias $=-.28 ; \mathrm{p}<.05)$.

Tabla 4. Estadísticos descriptivos para los niveles competitivos y valores $\mathrm{F}$

\begin{tabular}{|c|c|c|c|c|c|c|c|c|}
\hline & & \multicolumn{3}{|c|}{ Media } & \multicolumn{3}{|c|}{ Desviación típica } & \multirow[t]{2}{*}{$F$} \\
\hline & & $L$ & $A$ & $N$ & $L$ & $A$ & $N$ & \\
\hline \multirow{4}{*}{$I B D-R$} & Total & 2.00 & 2.00 & 1.97 & 0.44 & 0.41 & 0.46 & 0.33 \\
\hline & $A E$ & 1.81 & 1.89 & 1.89 & 0.61 & 0.58 & 0.61 & 0.65 \\
\hline & $R R P$ & 2.49 & 2.40 & 2.28 & 0.67 & 0.62 & 0.72 & $3.23^{*}$ \\
\hline & $D$ & 1.59 & 1.58 & 1.63 & 0.68 & 0.64 & 0.70 & 0.21 \\
\hline \multirow{4}{*}{$A B Q$} & Total & 2.20 & 2.20 & 2.14 & 0.56 & 0.55 & 0.60 & 0.41 \\
\hline & $A F E$ & 2.13 & 2.16 & 2.32 & 0.72 & 0.80 & 0.75 & 2.52 \\
\hline & $R S L$ & 2.48 & 2.45 & 2.17 & 0.73 & 0.70 & 0.64 & $8.36^{*}$ \\
\hline & $D P D$ & 1.98 & 1.97 & 1.91 & 0.80 & 0.86 & 0.88 & 0.22 \\
\hline
\end{tabular}

Burnout y volumen de entrenamiento. El volumen de entrenamiento se ha medido a través de las horas semanales de práctica y se ha fraccionado la muestra en 5 grupos en base a criterios estadísticos (agrupación de porcentajes obtenidos en las frecuencias): (1) deportistas que entrenan menos de tres horas y media semanales (14.7\% de los deportistas); (2) entre tres horas y media y siete horas y media (47.7\%); (3) a partir de siete horas y media hasta doce horas y media (24.3\%); (4) entre doce horas y media y dieciocho horas (6.6\%); (5) más de dieciocho horas semanales $(5.7 \%)$. Se han encontrado di- ferencias significativas en las dos subescalas que miden agotamiento: Agotamiento Emocional (IBD-R) y Agotamiento Físico/Emocional (ABQ). Las comparaciones a posteriori mediante la prueba de Scheffé indican que para Agotamiento Emocional existen diferencias significativas entre el grupo 5 y el 1 (diferencia de medias $=.44 ; \mathrm{p}<.05$ ) y para Agotamiento Físico/Emocional entre el grupo 5 y el 2 (diferencia de medias $=.50 ; \mathrm{p}<.05)$. Para ambas dimensiones, se observan valores medios más altos para el grupo 5 (Tabla 5). 
Tabla 5. Estadísticos descriptivos según las horas de entrenamiento y valores F.

\begin{tabular}{|c|c|c|c|c|c|c|c|c|c|}
\hline & & \multicolumn{4}{|c|}{$I B D-R$} & \multicolumn{4}{|l|}{$A B Q$} \\
\hline & & Total & $A E$ & $R R P$ & $D$ & Total & $A F E$ & $R S L$ & $D P D$ \\
\hline \multirow{5}{*}{ Media } & Hasta 3.50 horas & 1.20 & 1.76 & 2.51 & 1.60 & 2.25 & 2.16 & 2.52 & 2,08 \\
\hline & $3.50-7.50$ & 1.98 & 1.82 & 2.40 & 1.60 & 2.17 & 2.10 & 2.42 & 1,99 \\
\hline & $7.50-12.50$ & 1.99 & 1.91 & 2.34 & 1.59 & 2.10 & 2.28 & 2.20 & 1,81 \\
\hline & $12.50-18$ & 1.96 & 2.01 & 2.20 & 1.54 & 2.30 & 2.48 & 2.39 & 2,03 \\
\hline & Más de 18 horas & 2.12 & 2.20 & 2.34 & 1.70 & 2.27 & 2.60 & 2.30 & 1,90 \\
\hline \multirow{5}{*}{ Desv. Tip. } & Hasta 3.50 horas & 0.43 & 0.56 & 0.71 & 0.64 & 0.62 & 0.83 & 0.75 & 0,85 \\
\hline & $3.50-7.50$ & 0.42 & 0.58 & 0.65 & 0.68 & 0.57 & 0.74 & 0.72 & 0,87 \\
\hline & $7.50-12.50$ & 0.44 & 0.61 & 0.68 & 0.63 & 0.54 & 0.77 & 0.59 & 0,81 \\
\hline & $12.50-18$ & 0.47 & 0.79 & 0.56 & 0.57 & 0.61 & 0.77 & 0.76 & 0,98 \\
\hline & Más de 18 horas & 0.54 & 0.56 & 0.70 & 0.88 & 0.46 & 0.63 & 0.76 & 0,70 \\
\hline$F$ & & 0.64 & $3.34^{*}$ & 1.34 & 0.22 & 1.34 & $4.11^{*}$ & 2.82 & 1.28 \\
\hline
\end{tabular}

Nota. ${ }^{*}$ Significatividad estadística a nivel $\mathrm{p}<.05$

\section{Discusión y conclusiones}

En primer lugar cabe destacar que haber dispuesto de una muestra representativa de una variedad de deportes, nos permite plantear algunas cuestiones de futuro con cierta solidez, teniendo siempre presente que se trata de un trabajo de investigación que encuadra en una línea más ambiciosa que nos permita, en un futuro, aclarar algunos de los aspectos que nos pueden hacer comprender mejor el síndrome.

Quizás el dato más relevante es el que hace referencia al hecho de que cerca del $4 \%$ de los deportistas padece burnout. Este dato es muy significativo ya que estudios anteriores cifraban la prevalencia en porcentajes superiores (Garcés de Los Fayos, 1999; Jiménez et al., 1995; Medina y García, 2002). Quizás, la mejora de los instrumentos de evaluación, pueda explicar esta disminución ya que los datos han sido similares en ambos instrumentos, lo cual nos hace pensar que efectivamente dicha prevalencia es acorde a la realidad. Este $4 \%$, si se suman aquellos deportistas que presentan un alto riesgo de padecer el trastorno, sitúa el porcentaje en algo más del $15 \%$. Estos resultados se confirman si además consideramos que los datos de individuos afectados por algunas de las tres dimensiones que configuran el síndrome, de una manera evidente o presentando alto riesgo, y evaluadas por cualquiera de los dos instrumentos de medida, ofrecen porcentajes que no bajan del 14\%. Sin duda estos datos apuntan, al menos desde nuestra perspectiva, tres líneas de actuación de cara a un futuro inmediato: en primer lugar, profundizar en la mejor de los mecanismos de evaluación que permitan cifrar de una manera más segura el nivel de incidencia del síndrome; en segundo lugar, mejorar los instrumentos de evaluación, en el continuo, evaluación-intervención nos garantiza niveles óptimos de trabajo en el entrenamiento psicológico cotidiano; y, en tercer lugar, una mejora en la evaluación es sinónimo de mayor precisión en el diagnóstico diferencial que vuelve a incidir en la mejor actuación tanto en prevención como en intervención del síndrome.

Un aspecto paralelo que nos obligará a profundizar más en el estudio de burnout, es el hecho de que la Despersonalización, evaluada por el IBD-R, o Devaluación de la Práctica Deportiva, evaluada por el ABQ, pero ambas analizando un mismo conjunto de sentimientos y sensaciones de la persona que lo sufre (distanciamiento emocional), es la que más padecen los deportistas, superando el 5\%, confirmándose que es ésta quizás la variable esencial sobre la que hay que articular el conjunto de trabajos de investigación que pretende conocer el origen y desarrollo del burnout.

Por otra parte, y en el intento de comprender mejor ese origen y desarrollo del síndrome, nuestros resultados aportan aspectos tan interesantes como el hecho de que sean las mujeres las que se agotan más, en consonancia con otros trabajos (Reynaga, 2008, 2009; Reynaga y Pando, 2005). quizás porque la sobrecarga habitual en este género se incrementa con un esfuerzo evidente por el desarrollo deportivo buscado, y los hombres son los que muestran mayores niveles de despersonalización, evidenciando que quizá los hombres se muestren más distantes emocionalmente hacia las demás personas de su entorno deportivo.

También comprobamos que los deportistas con un nivel deportivo de carácter nacional/internacional presentan una sensación de realización personal y de logro inferior a los que tienen un nivel competitivo inferior, quizás porque los primeros ven más complicada la realidad que supone lo difícil que es llegar a estar entre los mejores, aspecto que no analizan todavía los que tienen un nivel competitivo inferior. Quizás, esto estaría en consonancia con los pensamientos distorsio- 
nadores que señalaban los estudios colombianos de Sierra y Abello (2008) e incluso con los componentes motivacionales de la investigación mexicana de Percastre (2009).

La edad también resulta relevante, desde el momento en que no existen diferencias significativas entre edades diferentes, lo que nos hace pensar que el síndrome puede afectar por igual en cualquier edad tal y como planteaba Feigley (1984).

Por último, los deportistas que más entrenan son los más propensos a sufrir agotamiento, tanto físico, medido a través del ABQ, como emocional, evaluado a través de los dos instrumentos. Es evidente que al esfuerzo físico hay que sumar el esfuerzo mental que supone el sacrificio por intentar lograr algo que no siempre se consigue, el éxito deportivo (Arce et al, 2012; De Francisco et al, 2009; Garcés de Los Fayos, 2013).

Los trabajos existentes en nuestro entorno más cercano, cultural e idiomáticamente, nos llevan a apostar por esta línea de investigación, si bien las estrategias han de guiar la investigación hacia el diseño y desarrollo de Programas de Actividad Física y Actuación Psicológica que incidan en la salud del deportista, incluso desde una perspectiva transnacional como plantean Garcés de Los Fayos y Medina (2002). En la búsqueda de detectar adecuadamente el síndrome e implementar actuaciones de prevención concretas, en el futuro habría que incidir especialmente en:

- Seguir ahondando en nuevos estudios epidemiológicos del síndrome en deportistas, analizando las posibles diferencias respecto al tipo de modalidad deportiva practicada (individual y colectiva) y clarificar los posibles diferencias entre categorías deportivas.

- Analizar las causas que ocasionan y desarrollan el síndrome, incorporando otras variables que pudiesen incidir como lesiones, personalidad, etc.

- Consolidar los instrumentos de medida que se muestran sensibles a la detección de este síndrome.

- La relación existente entre el elevado riesgo de padecer este síndrome y la esencia del deporte: el éxito en el mismo.

\section{Referencias}

1. Arce, C., De Francisco, C., Andrade, E., Seoane, G. y Raedeke, T. D. (2012). Adaptation of the Athlete Burnout Questionnaire in a Spanish Sample of Athletes. Spanish Journal of Psychology, 15, 1529-1536.

2. De Francisco, C., Arce, C., Andrade, E., Arce, I. y Raedeke, T. (2009). Propiedades preliminares de la versión española del Athlete Burnout Questionnaire en una muestra de jóvenes futbolistas. Cuadernos de Psicología del Deporte, 9, 45-56.

3. Dosil, J. (2008). Psicología de la actividad física y el deporte. Madrid: McGraw Hill.

4. Fender, L. K. (1989). Athlete burnout: Potential for research and intervention strategies. The Sport Psychologist, 3, 63-71.

5. Feigley, D. A. (1984). Psychological burnout in high-level athletes. Physician and Sportsmedicine, 12, 109-119.

6. Flippin, R. (1981). Burning out: When fast, young runners experience "failure" it can lead to self-destruction. Runner, 3, 76-78; 80-83.

7. Garcés de Los Fayos, E. J. (1999). Burnout en deportistas: un estudio de la influencia de las variables de personalidad, sociodemográficas y deportivas en el sindrome (tesis doctoral no publicada). Universidad de Murcia, Murcia.

8. Garcés de Los Fayos, E.J. (2004). Burnout en deportistas. Madrid: EOS

9. Garcés de Los Fayos, E. J., De Francisco, C y Arce, C. (2012). Inventario de Burnout en Deportistas Revisado (IBD-R). Revista de Psicología del Deporte, 21, 271-278.

10. Garcés de Los Fayos E. J., Jara, P. y Vives, L. (2006) Deporte y burnout. En E. J. Garcés de Los Fayos, A., Olmedilla y P. Jara, (coord.). Psicología $y$ deporte (pp. 525-533). Murcia: Diego Marín.

11. Garcés de Los Fayos, E. J. y Medina, G. (2002). Principios básicos a aplicar en el desarrollo de programas de intervención y prevención en deportistas con el síndrome de burnout. Propuestas desde una perspectiva Transnacional. Revista de Psicología del Deporte, 11, 259-267.

12. Garcés de Los Fayos, E.J., Olmedilla, A. y Jara, P. (2006). Psicología y deporte. Murcia. Diego Marín.

13. Gould, D., Tuffey, S., Udry, E. y Loerh J. (1996). Burnout in competitive junior tennis players: II. Qualitative Analysis. The Sport Psychologist, 10, 341-366.

14. Hernández, L. D. (2009). La presencia del sindrome de burnout en un equipo masculino de deportistas clase élite de ligas menores, quienes participan en el Deportivo Saprissa (tesis de Grado no publicada). Universidad Fidelitas de Costa Rica, Costa Rica.

15. Jiménez, G., Jara, P. y García, C. (1995). Variables relevantes en el contexto deportivo y sindrome de burnout. Presentado en el V Congreso Nacional de Psicología de la Actividad Física y el Deporte, Valencia.

16. Maslach, C. y Jackson S.E. (1981). MBI: Maslach Burnout Inventory. Manual. Palo Alto, CA: Consulting Psychologists Press.

17. Marín, E., Ortín, F., Garcés de Los Fayos, E.J. y Tutte, V. (2013). Análisis bibliométrico de burnout y optimismo en deporte. SportTK, 2(2), 77-87.

18. Medina G. (2001). Sindrome de Burnout y Locus de Control en deportistas cubanos de alto rendimiento (tesis de Licenciatura no publicada). Universidad de la Habana, La Habana.

19. Medina, G. y García, F. E. (2002). Burnout, locos de control y deportistas de alto rendimiento. Cuadernos de Psicología del Deporte, 2, 29-42.

20. Percastre, R. (2009). Análisis de la relación entre el sindrome de burnout $y$ los estilos de afrontamiento en deportistas de diferentes disciplinas: Caso Estado de México (memoria Diploma Estudios Avanzados). Universidad de León, León.

21. Raedeke, T. D. y Smith, A. (2001). Development and preliminary validation of an athlete burnout measure. Journal of Sport and Exercise Psychology, 23, 281-306.

22. Reynaga, P. (2008). Presencia de trastorno psicológico y burnout en jóvenes deportistas jaliscienses de alto nivel. Presentado en el II Congreso de la Sociedad Iberoamericana de Psicología de la Actividad Física y del Deporte, Torrelavega, Cantabria.

23. Reynaga, P. (2009). Prevalencia del síndrome de agotamiento crónico ("burnout") en jóvenes deportistas de alto rendimiento de Jalisco. Presentado en el Primer Encuentro on line de Psicología del Deporte de la SIPD, México.

24. Reynaga, P. y Pando, M. (2005). Relación del síndrome de agotamiento crónico (burnout), con el trastornos psicológico potencial en jóvenes deportistas. Investigación en Salud, 7, 153-160.

25. Sierra, C. A. y Abello, R. (2008). Burnout y pensamientos irracionales en deportistas de alto rendimiento. Psychologia, 2, 49-78. 
26. Tutte, V. (2009). Riesgo de abandono deportivo en jóvenes futbolistas (tesis doctoral no publicada). Universidad Autónoma de Barcelona, Barcelona.

27. Tutte, V., Blasco. T. y Cruz, J. (2006). Evaluación de los índices de burnout en un equipo femenino de baloncesto. Cuadernos de Psicología del Deporte, 6, 21-36Tutte, V. y Garcés de Los Fayos, E. J. (2012). Bur- nout en Iberoamérica: líneas de investigación. Cuadernos de Psicología del Deporte, 10, 47-56.

28. Tutte, V. y Suero, M. (2009). Compromiso Deportivo, juicio de control y burnout en dos equipos deportivos femeninos. Ciencias Psicológicas, III, 17-28. 